\title{
Relevance and usefulness of head CT-scan requests in the management of adult headaches: $A$ cross-sectional study in two referral hospitals in Cameroon
}

\section{Maggy Mbede ( $\sim$ maggymbede@yahoo.fr)}

Universite de Yaounde I Faculte de Medecine et des Sciences Biomedicales https://orcid.org/00000001-5538-9699

\section{Callixte Kuate Tegueu}

Universite de Douala Faculte de Medecine et Sciences Pharmaceutiques

\section{Ulrich Igor Mbessoh Kengne}

Université de Yaoundé 1 Faculté de médecine et des sciences biomédicales

\section{Pierre Ongolo Zogo}

Universite de Yaounde I faculte des sciences biomedicales et Medical

\section{Boniface Moifo}

Université de Yaoundé 1 Faculté de médecine et des sciences biomédicales

\section{Research article}

Keywords: relevance, usefulness, head CT-scan requisitions, headache, imaging referral guidelines, radioprotection, French Society of Radiology, Cameroon

Posted Date: September 27th, 2019

DOI: https://doi.org/10.21203/rs.2.14218/v1

License: (c) (i) This work is licensed under a Creative Commons Attribution 4.0 International License. Read Full License 


\title{
Relevance and usefulness of head CT-scan requests in the management of adult headaches: A cross-sectional study in two referral hospitals in Cameroon
}

\author{
1- MBEDE Maggy ${ }^{(1,2)}$ : maggymbede@yahoo.fr \\ 2- KUATE TEGUEU Callixte ${ }^{(1,3)}$ : kuate.callixte $@$,gmail.com \\ 3- MBESSOH KENGNE Ulrich Igor ${ }^{(1)}$ : mbessohulrich@yahoo.com \\ 4- ONGOLO ZOGO Pierre ${ }^{(1,2)}$ : pc.ongolo@gmail.com \\ 5- MOIFO Boniface ${ }^{(1,4)}$ : bmoifo@yahoo.fr
}

(1): Faculty of Medicine and Biomedical Sciences - University of Yaounde 1 (Cameroon)

(2): Radiology and medical imaging unit, Central Hospital of Yaounde (Cameroon)

(3): Neurology department, Laquintinie Hospital of Douala (Cameroon)

(4): Radiology department, Yaounde Gynaecology-Obstetric and Pediatric Hospital (Cameroon)

Corresponding author: MBEDE MAGGY

Email: maggymbede@yahoo.fr, maggymbede@gmail.com,

\begin{abstract}
Background: In developing sub-Saharan Africa countries such as Cameroon, the head CT-scan, which is more available and accessible than magnetic resonance imaging, gold standard, exposes to a high irradiation dose level and is still expensive. In those countries, head CT-scan has become a routine exploration in patients with headache. In front of this inflation, it is necessary to assess CT-scan requests and their profitability in the patient management.

Objective: To assess the relevance and the usefulness of head computerized tomography scanner (CTscan) requests in the management of adult headache in two referral teaching hospitals of Cameroon, in order to improve their appropriateness.

Methods: This was a prospective cross-sectional study conducted in medical imaging units of the Central Hospital of Yaounde and the Laquintinie Hospital of Douala on five months period. We confronted indication mentioned on head CT-scan requests to the recommendation grades and indication's mentions of the referral imaging guidelines of the French Society of Radiology in order to assess the relevance on the one hand. We also confronted head CT-scan results to the expectations of the requesting physicians in order to assess the usefulness on the other hand.
\end{abstract}

Data analysis: Non-parametric statistical analysis.

Results: During the study period, 169 examinations requests for headaches were collected. Symptom was the main clinical data mentioned on CT-scan requests (60.4\%), followed by the suspected clinical diagnosis (40.2\%). Requesting physicians were general practitioners $(50.3 \%)$, followed by neurologists in $14.2 \%$. Overall, $92.3 \%$ of requests were judged appropriate while $2.4 \%$ were non-indicated. The general practitioner's requests were non-indicated in $75 \%$ of the cases. Inappropriate requests multiplied by two the risk of having no significant abnormality on the head CT-scan. Of all the head CT-scan requests, $73.2 \%$ were useful and $100 \%$ of relevant CT-scans were also useful. $45.5 \%$ of useless head CT-scans were requested by a general practitioner.

Conclusion: Physicians need to follow the guidelines and justify the use of head CT-scans in patients suffering from headache in order to improve the relevance and usefulness of head CT-scans requests.

Keywords: relevance, usefulness, head CT-scan requisitions, headache, imaging referral guidelines, radioprotection, French Society of Radiology, Cameroon. 


\section{Background}

Headache or cephalalgia is defined as diffuse pain in various parts of the head, with the pain not confined to the area of distribution of a nerve [1]. Headache is among the most common pain problems encountered in daily practice [1-3]. International Headache Society classifies headache into primary (without any organic cause) or secondary (with organic cause) $[1,4,5]$. In most instances, the physician can accurately diagnose a patient's headache and determine whether neuroimaging is indicated or not [1].

The indication refers to a description of a patient clinical's state on an examination request, using either symptom, clinical sign, syndrome, clinical diagnosis, or the aim of the examination [6]. Each of them can be associated to the past history summary or the information on the evolution of causal pathology [6]. Additionally, the indication is an obligation for the justification of the examination, which serves as a guide for the exam achievement, the interpretation, and consequently improve the sensitivity [6].

Owing to the complexity, the multiplicity, and the heterogeneity of clinical presentations, the use of neuroimaging for headaches is frequent and well codified. Additionally, referral guidelines for medical imaging, such as the one of the French Society of Radiology, have been elaborated for physicians in order to help them decide when an imaging study would be useful and identify the most appropriate examination for a particular patient in term of radioprotection [7]. This guideline assesses the relevance of an imaging examination according to six indication mentions. Thus, the examination can be indicated, indicated only in specific cases, specialised (must be requested only by specialist physicians), initially non-indicated, non- indicated, and contraindicated [7]. Four recommendation's grades (A, B, C, AE), based on decreasing scientific evidence levels, have been proposed for each imaging examination, in order to bring out both diagnosis sensitivity in given clinical situation and clinicians-radiologists expectations [6,7]. Indeed, neuroimaging in adult headache is usually requested by specialists, resident students and general physicians. The result is useful, either positive or negative, when it has a substantial impact on the patient management and care $[6,8]$.

In developing countries of sub-Saharan Africa such as Cameroon, the head CT-scan, which is more available and accessible than magnetic resonance imaging (IRM), gold standard [1], exposes to a high irradiation dose level [9] and is still expensive. Also, head CT-scan has become a routine exploration in patients with neurological clinical presentation [10] such as headache. In front of this inflation, it is necessary to assess CT-scan requests and their profitability in the patient management $[10,11]$.

To our knowledge, no study assessing head CT-scan requests for adult headache disorder has been carried out in Cameroon. Therefore, we initiated to assess the relevance and the usefulness of head CTscan requests in the management of adult headache's in two referral hospitals in Cameroon, in order to improve their appropriateness.

\section{Methods}

\section{Design}

We conducted a prospective cross-sectional study in medical imaging units of the Central Hospital of Yaounde and the Laquintinie Hospital of Douala which are two teaching hospitals in Cameroon, for a period of five months, from January to May 2017.

\section{Sampling}

We consecutively and non-exhaustively included head CT-scan requests, with or without contrast enhancement, of patients aged from 18 years or above, sent for the investigations of headache. Headache could be either the single symptom mentioned on the request or associated to others various clinical data such as symptoms, syndromes, clinical diagnosis, examination finality, and past history. All included requests were sent from those teaching hospital's units. The minimal sample size was calculated using the formula $n=t^{2} \times p(1-p) / m^{2}$ where $n$ is the minimal sample size required for the study, $t$ is the accuracy level to $95 \%$ (standard value is 1.96), $p$ is the estimated prevalence in the study setting, $m$ is the error margin to 5\% (standard value is 0.05). We referred to the study of Becker LA and al, title Use of CTscan for the investigation of headache, which found that CT-scans were ordered for approximately $3 \%$ of patients with headaches $[1,12]$. The minimal sample size was therefore estimated based on that prevalence at 45 head CT-scan requests for headache. 


\section{Data collection and statistical analysis}

After informed consent of eligible patients, data were collected on a pre-established data sheet and made anonymous before any computerization. Clinical information contained on the request were classified as symptom, syndrome, examination finality, clinical diagnosis, and past history. Then, they were confronted with indication's mentions (indicated, indicated only in specific cases, specialised, nonindicated, and contraindicated) of the referral guidelines for medical imaging of the French Society of Radiology, and to the four recommendation grades (A, B, C, AE) of an imaging examination. The indication's mentions were equally confronted with the physician qualification. After collecting clinical information on the request, the head CT-scan was performed in helical mode (HITACHI SUPRIA 16 slices) with or without contrast enhancement according to clinical situation. The CT-scan reading was performed in both cerebral and bone windows by at least five years' experience radiologists in head CTscan reading. The results were classified into two groups (normal and abnormal) and four results (positive without associated lesion, positive with associated lesion, negative and abnormal, negative and normal) related to the expectations of requesting physicians. We have considered positive without associated lesion, a head CT-scan result revealing the significant lesion as clinically suspected by the requesting physician with no other lesion. The result was positive with associated lesion when the head CT-scan showed the lesion as clinically suspected, associated with one or more other significant lesions. The result was negative and abnormal when the clinically suspected lesion was not found, but the CTscan showed one or more other significant lesions. Therefore, a request was considered useful when its result was either positive with and without associated lesions, or negative and abnormal. The results of head CT-scan were equally confronted with both indication mentions and physician qualification.

Statistical analysis has been performed using the SPSS version 23.0 software. The Chi-square test was used for the percentages comparison, and the Odds ratios were used to bring out associations. A p-value less than 0.05 was considered as statistically significant.

\section{Results}

During our study period, 169 CT-scan requests for headache were collected.

The symptom was the most prevalent clinical data mentioned on CT-scan requests $(60.4 \%)$, followed by clinical diagnosis (40.2\%). A same request could have one or more clinical information (figure 1).

Head CT-scan requests were mainly done by general practitioners $(50.3 \%)$, followed by neurologists (14.2\%). According to referral imaging guideline of the French Society of Radiology, 92.3\% of requests were appropriately prescribed $(34.9 \%$ were indicated, $53.3 \%$ were indicated only in specific cases and $4.1 \%$ were specialised). Four head CT-scan requests were non-indicated (2.4\%) and three of them were requested by a general practitioner (75\%) (Table 1);

The most head CT-scans requested were of grade A and B evidence recommendation (92.9\%) (Table 2).

The table 3 confronts indication's mention to the results of the CT-scan.

$73.2 \%$ of head CT-scans requested were useful. All indicated CT-scans were also useful (100\%), and twenty-six of thirty-seven CT-scans indicated only in specific cases were useful (70.3\%).

On table 4, we noticed that inappropriate requests (initially non-indicated and non-indicated) multiplied by two the risk of having no significant abnormality on the head CT-scan.

The table 5 confronts the result of the CT-scan to the physician qualification. Of eleven useless head CT-scan, five $(45.5 \%)$ were requested by a general practitioner.

\section{Discussion}

The justification of medical imaging examinations is the first basic radioprotection principle of patients exposed to ionizing radiations $[9,12,13]$.

Radiology request are an essential communication tools used by clinicians referring patients for radiological investigations. The clinician is required to state the reason for referral as this helps radiologists to better understand the patient's condition [14]. This reason can be mentioned as a symptom, syndrome, clinical diagnosis suspected, finality of the examination, and/or notable past 
history of the patient. In our study, the main clinical information on requests was the symptom, headache, in $60.4 \%$ of the requests.

The study conducted by Moifo and al in Cameroon on the relevance of indications of medical imaging examinations showed different results with the symptom as main clinical information on the requests in $30.9 \%$ of their cases[6]. That study included additionally to CT-scan, the radiography, the ultrasonography. The participation of those additional examinations could explain the difference observed in both proportions. Symptom and other clinical information written on the requests are also determinant on the justification, the relevance of the indication of the examination, and lead the choice of the best neuroimaging technique to perform according to patient's clinical condition and international evidence-based guidelines.

Therefore, we found that $34.9 \%$ of head CT-scans performed for headache were indicated, according to the appropriateness guidelines for medical imaging explorations of the French Society of Radiology. This proportion is less than 59.5\% found by Moifo and al [6]. Indeed, CT-scans represented only 13\% of their sampling. Evolution of technical and practices in neuroradiology is ascertained [11], leading to frequent readjustments in international guidelines for medical imaging [7]. So, the advent of MRI (gold standard) has substantially decreased head CT-scan indications and requests for neurological disorders, which is therefore mostly indicated only in specific cases. We found that $53.3 \%$ of head CT-scans requested for headache disorder were indicated only in specific cases, probably due to the expensiveness and low availability of MRI in our environment.

General practitioners ordered $50.3 \%$ of head CT-scan, followed by neurologists with $14.2 \%$ of requests. You and al found in Canada that $75.9 \%$ of head CT-scans requested for headache condition were the ordering of general practitioners, followed by neurologists with $15.6 \%$ of requests [8]. This reality illustrates the status of general practitioners in first line of patient's care process in health care systems. They ensure the selection of emergency cases, and subsequently if needed they refer the patients to specialist physician after initial check-up with clinical and paraclinical tools.

Additionally, in teaching hospital setting, some requests of general practitioners are written from the order of specialist physicians.

In spite of this high level of requests ordered by general practitioner, the frequency of non-indicated CTscans is $2.4 \%$ in our study. This is near to $1.5 \%$ found by Moifo and al [6]. This low prevalence firstly denotes the improvement of physicians' knowledge on compatibility neuroimaging-clinical condition like described in guidelines, and secondly the ability of radiologists to reject performing imaging when it is not justified according to the clinical condition considered. Indeed, we found that inappropriate head CT-scan for headache significantly increases the risk to find no abnormality, and three of four nonindicated requests were ordered by general practitioner $(75 \%)$ as they requested most head CT-scans in adults with headache.

Technical developments of CT-scan imaging, especially the increase of the avaibility of multislice CTscan, with increased scanning speed and volume as well as higher spatial and temporal resolution, have significantly enhanced the diagnostic value of CT-scan in many clinical applications [11]. CT-scan has become an important diagnostic imaging modality, with high diagnostic accuracy and efficacy in both traumatic and non-traumatic conditions [11].

Justification of the application of CT-scan is one of the main principles that physicians need to be aware of when choosing CT-scan as the first-line technique for diagnosis. It has been reported that $30 \%$ or more of the CT-scans currently performed may be unnecessary [11]. This report corroborates our results, with $27 \%$ of CT-scans ordered in patients with headache and which didn't impact on patient management. Additionally, Jordan et al recently reported that CT-scan imaging of non-focal headache in emergency settings has limited cost efficacy due to a lower percentage of positive clinically significant results. $31.8 \%$ positive CT-scan findings were found in their study group, but only $1.02 \%$ showed clinically significant results which required a change in patient management [15].

There is also a relationship between a justified CT-scan and his value and contribution in the patient management. $100 \%$ of indicated CT-scans and $70.3 \%$ of head CT-scans indicated only in specific cases were useful in our study group. Renaud Emont and al showed that $97.5 \%$ of CT-scans with normal result were not useful for the patient's management [10]. This study show that a better ordering of requests could reduce significantly inappropriate CT-scans [10]. 


\section{Conclusion}

Since CT-scan imaging of headache disorder has become a widespread and growing problem with significant economic implications, physicians need to follow the guidelines and justify the use of head CT-scan in order to improve his relevance, usefulness, and profitability for the patient management.

\section{List of abbreviations}

CT-scan $=$ Computerized tomography scanner

MRI= Magnetic resonance imaging

$\mathrm{GP}=$ General practitioner

ENT $=$ Ear-Nose-Throat specialist

\section{Declarations}

\section{Ethics approval and consent to participate}

This research has been approved by the ethics review committee of the faculty of medicine and biomedical sciences - University of Yaoundé 1, by the ethics review committee of the Yaounde central hospital and the Laquintinie hospital of Douala.

All the patients signed their informed consent before been enrolled in the study.

\section{Availability of data and materials}

The data and materials are available on files attached.

\section{Competing interests}

The authors declare have no conflict of interests in relation to this article.

\section{Fundings}

No funding was received for this study.

\section{Author's Contributions}

- Conception and design of the study: Moifo Boniface, Mbede Maggy, Mbessoh Kengne Ulrich Igor, Ongolo Zogo Pierre

- Data collection: Mbessoh Kengne Ulrich Igor, Kuate Tegueu Callixte

- Data analysis: Mbessoh Kengne Ulrich Igor, Mbede Maggy

- Data interpretation: Mbede Maggy, Mbessoh Kengne Ulrich Igor

- Article drafting: Mbede Maggy, Mbessoh Kengne Ulrich Igor

- Article revising: Mbede Maggy, Ongolo Zogo Pierre, Moifo Boniface

- Final approval: Mbede Maggy, Mbessoh Kengne Ulrich Igor, Kuate Tegueu Callixte, Ongolo Zogo Pierre, Moifo Boniface

\section{Acknowledgements}

We are grateful to:

- The staff of medical imaging departments of the Central Hospital of Yaounde and the Laquintinie Hospital of Douala for their collaboration

- The administration of the Central Hospital of Yaounde and the Laquintinie Hospital of Douala for their both authorization.

\section{References}

1. Randall Clinch C, Edward HF. Evaluation of Acute Headaches in Adults. Am Fam Physician. 2001;63(4):685-92. https://www.aafp.org/afp/2001/0215/p685.html

2. Fromont A, Ben Salem D, Moreau T, Ricolfi F, Giroud M. Acute headaches. EMC- Medicine. 2005;2(1):66-81.

3. Valin A. Head CT-scan in the exploration of non-traumatic acute headaches at the emergency department: confrontation of practice with data from the literature. [Thesis]. Paris 6 University; 2014. 
M Mbede and al.

4. Alsadig Tarig MS. Evaluation of chronic headache in Sudanese patients by using computed tomography [Thesis]: Sudan University of Science and Technology; 2016. http://repository.sustech.edu/handle/123456789/15641

5. Aygun D, Bildik F. Clinical warning criteria in evaluation by computed tomography the secondary neurological headaches in adults. Eur J Neurol. 2003;10(4):437-42.

https://doi.org/10.1046/j.1468-1331.2003.00645.x

6. Moifo B, Ndeh Kamgnie M, Ninying Fuh F, Zeh OF, Tebere H, Moulion Tapouh JR, and al. Pertinence of indications of medical imaging examinations at Yaounde - Cameroon. Health Sci Dis. 2013;14(4). http://hsd-fmsb.org/index.php/hsd/article/view/246

7. French society of Radiology. Imaging referral guideline. Paris Ed 2013. http://www.gbu.radiologie.fr/.Accessed 02 Oct 2016.

8. You JJ, Gladstone J, Symons S, Rotstein D, Laupacis A, Bell CM. Patterns of Care and Outcomes After Computed Tomography Scans for Headache. Am J Med. 2011;124(1):58-63. e1. https://doi.org/10.1016/j.amjmed.2010.08.010

9. Brenner DJ, Hall EJ. Computed Tomography - An Increasing Source of Radiation Exposure. N Engl J Med. 357:2277-84. https://www.nejm.org/doi/full/10.1056/nejmra072149

10. Emont R. Pertinence of the prescription of head CT-scan at the emergency department. Diss 2005. $166 \mathrm{p}$.

11. Sun Z, Vijayananthan A. Is utilisation of computed tomography justified in clinical practice? Part I: application in the emergency department. Singapore Med J. 2010;51(3):200-6. https://espace.curtin.edu.au/handle/20.500.11937/36115

12. Doudenkova V, Bélisle-Pipon J-C. Overuse of medical imaging: A principle-based approach for a suitable justification of radiological examinations. Éthique Santé. 2015;12(4):225-33.

13. Tahvonen P, Oikarinen H, Pääkkö E, Karttunen A, Blanco Sequeiros R, Tervonen O. Justification of CT examinations in young adults and children can be improved by education, guideline implementation and increased MRI capacity. $\mathrm{Br}$ J Radiol. 2013;86(1029). https://www.birpublications.org/doi/abs/10.1259/bjr.20130337

14. Akinola R, Akinkunmi M, Wright K, Orogbemi O. Radiology request forms: are they adequately filled by clinicians? Internet J Radiol. 2009;12(1).

15. Jordan YJ, Johnson BL, John EJ. Computed Tomography Imaging in the Management of Headache in the Emergency Department: Cost Efficacy and Policy Implications. J Natl Med Assoc. 2009;101(4):331-5. https://doi.org/10.1016/S0027-9684(15)30880-4

\section{Figure Legend}

Figure 1: Types of clinical information on CT-scan requests

Table 1: Confrontation between physician qualification and indication's mention

Table 2: Confrontation between indication's mention and significant abnormal CT-scan finding

Table 3: Confrontation between head CT-scan results and the indication's mention

Table 4: Confrontation between recommendation grades and the types of clinical information

Table 5: Confrontation between result modality and physician qualification 
Figures

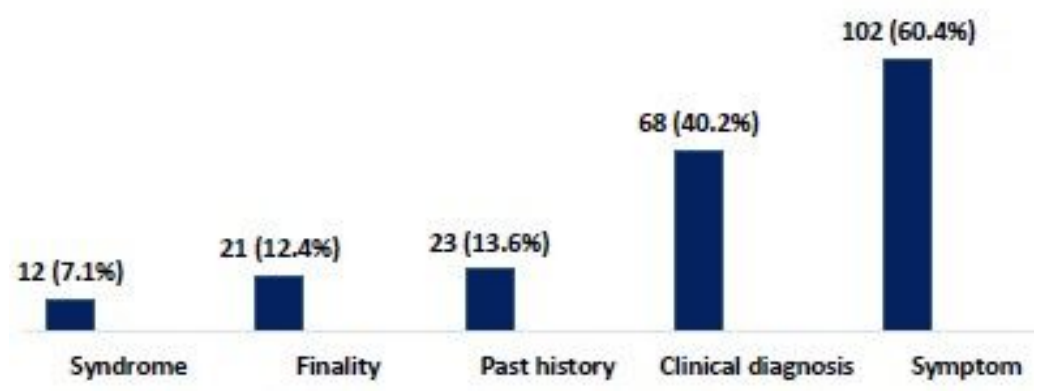

Figure 1

types of clinical information on CT-scan requests 